\title{
Führung II: Kontext- und genderspezifische Bedingungsanalysen und die Entwicklung von Fördermodellen
}

\author{
Telse A. Iwers-Stelljes
}

Das vorliegende Heft ist wie das Heft zuvor (4.2011) dem Thema Führung gewidmet.

Im ersten Heft wurden zunächst mitarbeiterförderliche Aspekte von Führung in den Blick genommen. Dabei wurde von Sebastian Sauer, Karin Andert, Niko Kohls und Günter F. Müller untersucht, inwieweit achtsame Führung gesundheitsförderlich sein kann. Ebenso wurden von Jenny Rohde, Sylvie Vincent und Monique Janneck kompetenz- und karriereförderliche Verhaltensweisen von Führungskräften in einer Interviewstudie exploriert. Im Anschluss an diese empirischen Bedingungsanalysen wurden zwei Transferierungen präsentiert: einerseits ein Konzept der Führung durch Selbstführung (Günter F. Müller, Martin Sauerland, Bianca Butzmann) und andererseits ein Modell der Entwicklung von Führungskompetenz durch die Verschränkung von Selbstreflexion und Methodenkenntnis (Telse Iwers-Stelljes).

Allen Beiträgen gemeinsam ist, dass sie auf die Wechselwirkung von Führung und MitarbeiterInnen fokussieren und damit die interindividuelle Bedeutung ebenso wie die gruppendynamische Relevanz der Führenden hervorheben.

Führungskräfte werden, so zeigten diese Beiträge zusammengefasst, gegenwärtig verstärkt aus der Perspektive der MitarbeiterInnenförderung beurteilt. Jeweilige Führungsstile werden dabei zunächst ausgehend von ihrem Maß an Dialogorientierung und Demokratieverständnis gemessen. Damit schließt die Analyse von Bedingungen gelungener Führung im weitesten Sinne an die schon von Kurt Lewin vorgelegten Interaktionsstile und deren Auswirkungen auf die jeweiligen geführten Gruppen an. Zugleich werden diese Aspekte differenziert erweitert. Aktuelle Konzepte der Wechselseitigkeit von Führung und MitarbeiterInnenverhalten liefern äußerst differenzierte Erkenntnisse zu verschiedenen facetteneichen Aspekten von Führung, die sich über Fragen der MitarbeiterInnenmotivation und -förderung, des MitarbeiterInnencoachings, der Konfliktreduktion und Gesundheitsförderung bis hin zu Fragen zur Entwicklung von Achtsamkeit erstrecken. Neu erscheint in diesem Zusammenhang auf der einen Seite die hohe Akzep-

Online publiziert: 16.12 .2011

(C) VS Verlag für Sozialwissenschaften 2011

T. A. Iwers-Stelljes $(\bowtie)$

Hamburg, Deutschland

E-Mail: GDO@iwers-stelljes.de 
tanz von gruppendynamischen ebenso wie subjektorientierten Kriterien in ökonomischen und technisch-naturwissenschaftlichen Kontexten sowie deren Ergänzung um weitere wesentliche Aspekte förderlichen Führungsverhaltens. Ebenso neu erscheint die Entwicklung von differenzierten genuin psychologisch und/oder pädagogisch konnotierten Transferkonzepten. Damit werden zunächst in bildungs-, erziehungs- und begleitungsorientierten Non-Profit-Bereichen entwickelte Kriterien gruppendynamisch orientierter und/oder subjektorientierter Leitung auf Felder übertragen, die sich in jüngerer Zeit verstärkt um Humanisierungsprozesse bemühen, zugleich aber in polarisierende Spannungslagen weltwirtschaftlicher Brisanz geraten. Diese dürfen bei allen ermutigenden Forschungsentwicklungen nicht aus dem Blick geraten. Sie finden ihren Niederschlag in zunehmender Stresswahrnehmung und im Belastungserleben von MitarbeiterInnen ebenso wie von Führenden und dies sicher auch aufgrund der kontroversen Anforderungen zwischen Subjekt- und Gesellschafts- sowie Marktorientierung.

Auf der anderen Seite zeigt sich, dass genuin in ökonomischen Profit-Kontexten eruierte und etablierte Modelle, die im weitesten Sinne verschiedenen Formen von Führung auf dem Hintergrund von Qualitätssicherung und Qualitätsentwicklung zugerechnet werden, auf Non-Profit-Bereiche transferiert werden. Auch mit dieser Wendung sind Spannungslagen verbunden, hier durch die Orientierung an Kriterien von Effizienz im Kontext von subjektorientierten Bedürfnissen der persönlichen Entwicklung in Beziehungen.

Insgesamt lassen beide Zuwegungen langfristig auf eine Konglomeration hoffen, bleiben derzeit allerdings noch mit verschiedenen feldabhängigen Kontroversen ausgestattet.

Das vorliegende zweite Heft zum Thema Führung ergänzt die Erkenntnisse der so als antinomisch zu bezeichnenden Anforderungslagen und erweitert die Perspektiven verschiedener Bedingungsanalysen und ableitbarer Fördermöglichkeiten von Führung. Dabei stehen zunächst klassische Handlungsfelder von Führung im Fokus, die mit innovativen Zugängen zur Reflexion und Entwicklung von Führungskompetenz ausgestattet werden, um vorfindbare Grundspannungen innovativ zu bewältigen und damit an die erste aufgezeigte Entwicklungslinie anzuschließen.

Diesen Bereich deckt die Arbeit von Yvette Voelschow ab, welche die Entwicklung und Evaluation eines kollegialen Coachings für Führungskräfte aus Polizei und Justiz beschreibt. Zugleich weist sie sich damit als Forschung an der Grenze der beiden oben skizzierten Bereiche aus, denn einerseits werden klar hierarchisierende Aspekte sichtbar, andererseits entbehrt der hier untersuchte Bereich einer eindeutigen Profit-Orientierung. Sie beschreibt eine Form kollegialen Coachings für Führungskräfte aus Polizei und Justiz anhand von Bedarfserhebungen zu den Dynamiken von Konkurrenz und Unterstützung innerhalb der Berufsgruppen (besonders bei der Polizei) und zwischen den Gruppen (Polizei und Justiz). Der Einfluss organisationsimmanenter bzw. organisationskultureller Aspekte spielt hier ebenso eine Rolle, wie der personenzentrierte Ansatz, mit dem der komplizierten Dynamik begrenzt aber stellenweise doch auch entgegengewirkt werden kann. Ihr Beitrag trägt den Titel: ,Kollegiales Coaching‘ in der Führungskräfteentwicklung des Landesdienstes'.

Im Anschluss wird verstärkt auf genderspezifische Fragestellungen von Führung fokussiert. Damit orientieren sich die Folgebeiträge an der Transformation von subjektorientierten Kriterien sozialisatorischer, psychologischer und pädagogischer Erkenntnis- 
genese in ökonomisch ausgewiesene Kontexte. So wird von Judith Oerding eine Studie zu den Anliegen weiblicher Führungsnachwuchskräfte in Mentoringprozessen vorgelegt. Ziel dieser Untersuchung war es, die Beratungsform Mentoring für weibliche Führungsnachwuchskräfte zu veranschaulichen und deren Bedeutsamkeit für berufliche Förderprozesse an Einzelfällen nachzuzeichnen. Ihr Beitrag trägt den Titel: ,Was bewegt weibliche Führungsnachwuchskräfte? - Typische Anliegen aus der Mentoringpraxis anhand von Fallgeschichten".

Daniela Rastetter und Doris Cornils berichten unter dem Titel ,Frauen und Führung: Networking als aufstiegsförderliche Strategie' von Ergebnissen aus einer Interviewstudie mit 25 männlichen und weiblichen Führungskräften bezüglich des mikropolitischen Handlungsfeldes Networking. Aus diesen Daten können die Autorinnen Erkenntnisse zum Zusammenhang von Mikropolitik, Gender und Aufstieg generieren.

Sylvie Vincent berichtet von einer Untersuchung zu gesundheits- und entwicklungsförderlichem Führungsverhalten unter genderspezifischer Perspektive. Auf Basis einer empirischen Untersuchung von 2000 MitarbeiterInnen wurde ein Modell gesundheitsund entwicklungsförderlicher Führung konzipiert und validiert. Ihr Beitrag trägt den Titel: ,Gesundheits- und entwicklungsförderliches Führungsverhalten: Gendertypische Differenzen'.

Den Thementeil dieses Heftes abschließend liefern Jenny Rohde, Sylvie Vincent und Monique Janneck unter dem Titel ,Kompetenz- und karriereförderliches Führungsverhalten: Zum Stand der Forschung' einen Überblick über aktuelle Literatur zu genderspezifischen Führungsaspekten, mit der die Breite der aktuellen Bearbeitungen dieser Perspektive ebenso verdeutlicht wird wie deren hohe Transferbedeutung unter besonderer Berücksichtigung des Zusammenschlusses von Konzeptionen profitorientierter wie nonprofit-orientierter Herkunft. 